TITLE:

\title{
Quantum dynamics study on predissociation of H-3 Rydberg states: Importance of indirect mechanism
}

\author{
$\operatorname{AUTHOR}(\mathrm{S})$ : \\ Tashiro, Motomichi; Kato, Shigeki
}

CITATION:

Tashiro, Motomichi ...[et al]. Quantum dynamics study on predissociation of H-3 Rydberg states: Importance of indirect mechanism. JOURNAL OF CHEMICAL PHYSICS 2002, 117(5): 2053-2062

ISSUE DATE:

2002-08-01

URL:

http://hdl.handle.net/2433/50519

\section{RIGHT:}

Copyright 2002 American Institute of Physics. This article may be downloaded for personal use only. Any other use requires prior permission of the author and the American Institute of Physics. 


\title{
Quantum dynamics study on predissociation of $\mathrm{H}_{3}$ Rydberg states: Importance of indirect mechanism
}

\author{
Motomichi Tashiro ${ }^{\mathrm{a})}$ \\ Department of Physics, Graduate School of Science, Kyoto University, Kyoto 606-8502, Japan \\ Shigeki Kato ${ }^{\text {b) }}$ \\ Department of Chemistry, Graduate School of Science, Kyoto University, Kyoto 606-8502, Japan
}

(Received 21 February 2002; accepted 14 May 2002)

\begin{abstract}
Predissociation of $\mathrm{H}_{3}$ Rydberg states was investigated using the effective Hamiltonian which describes the vibronic transitions among the Rydberg states as well as the predissociation through the vibrationally excited $2 s$ states. The motion of a Rydberg electron and the vibrations of the $\mathrm{H}_{3}^{+}$ ion core was treated simultaneously without assuming the Born-Oppenheimer approximation. We developed the effective potential for a Rydberg electron, which contains the Coulomb potential and the exchange effect. The energies and predissociation lifetimes of $\mathrm{H}_{3}$ Rydberg states were obtained by analyzing the effective Hamiltonian and compared with the available experimental values. The $s$ and $p$ Rydberg states with lower vibrational excitation have lifetimes between a few ps to $1 \mathrm{~ns}$ and show an irregular lifetime distribution with respect to the principal quantum number. In contrast, $d$ and $f$ Rydberg states have longer lifetime, $10 \mathrm{~ns}$ for example. The energy level spacings of the Rydberg states obey the distribution close to the Poisson one and thus indicates these states being regular. The route of predissociation was investigated by propagating a wave packet as well as analyzing the eigenvectors of the effective Hamiltonian. We found that the energy level matching between nearby states play an important role for efficient predissociation. The present results suggest that the predissociation of the $\mathrm{H}_{3}$ molecule and the dissociative recombination of the $\mathrm{H}_{3}^{+}$ion might be efficient under rotational excitation through inclusion of additional energy levels. (C) 2002 American Institute of Physics. [DOI: 10.1063/1.1490918]
\end{abstract}

\section{INTRODUCTION}

The triatomic hydrogen $\mathrm{H}_{3}$, the simplest polyatomic molecule, has been the subject of many experimental and theoretical studies for a long time. ${ }^{1-5}$ It also attracts the attention with respect to the dissociative recombination (DR) of the $\mathrm{H}_{3}^{+}$ion with an electron, which is one of the most important reactions in interstellar chemistry. The $\mathrm{H}_{3}^{+}$DR process starts with the capture of the incident electron to form the vibrationally excited $\mathrm{H}_{3}$ Rydberg states, and subsequent predissociation follows to produce $\mathrm{H}_{2}+\mathrm{H}$ or $\mathrm{H}+\mathrm{H}$ $+\mathrm{H}$ fragments. In order to describe this DR process, it is required to clarify the mechanism of nonadiabatic transitions among the bound Rydberg states as well as to the dissociative continua. In this respect, quantum dynamics studies of the predissociation of $\mathrm{H}_{3}$ Rydberg states are indispensable to understand the $\mathrm{H}_{3}^{+}$DR reaction. It is noteworthy that there are still discrepancies between the experiments and theoretical calculations on the magnitudes of the rate constants. ${ }^{6,7}$

For several $\mathrm{H}_{3}$ Rydberg states, the experimental investigations have been performed to determine the predissociation lifetimes, ${ }^{8}$ the branching ratio, ${ }^{9}$ the rovibrational distribution of product $\mathrm{H}_{2}$ in two-body decay, ${ }^{10-12}$ and the fragments momentum correlation in three-body decay. ${ }^{13,14}$ Among these various experimental quantities, the lifetimes

\footnotetext{
${ }^{a)}$ Electronic mail: tashiro@tap.scphys.kyoto-u.ac.jp

${ }^{b)}$ Electronic mail: shigeki@kuchem.kyoto-u.ac.jp
}

exhibit interesting property. The ground vibrational state for the $\mathrm{H}_{3} 2 s A_{1}^{\prime}$ Rydberg state predissociates very rapidly with the lifetime of 0.19 ps. ${ }^{15}$ The lifetime of the $3 s A_{1}^{\prime}$ state is longer than 66 ps. ${ }^{8,16,17}$ but for the $4 s$ and $5 s A_{1}^{\prime}$ states it becomes shorter, $7.6 \mathrm{ps}$ and $3.8 \mathrm{ps}$, respectively. ${ }^{8}$ For the $3 p E^{\prime}$ state, the lifetime is longer than $66 \mathrm{ps}$ while the $4 p E^{\prime}$ state shows a faster rate. ${ }^{8}$ This behavior, i.e., the increase of the rate constant with increasing the principal quantum number, seems irregular, since the direct coupling between the Rydberg and dissociative $2 p E^{\prime}$ states becomes smaller for larger principal quantum number, in general. One of the possible interpretations is the indirect mechanism, ${ }^{18}$ where the $4 s$ and $5 s$ Rydberg states vibronically couple with the vibrationally excited $2 s A_{1}^{\prime}$ states whose decay rates are large.

Theoretical studies of higher Rydberg states have been advanced based on the multichannel quantum defect theory (MQDT), which provide a wealth of information on the coupling between the continuous and bound electronic states of $\mathrm{H}_{3} .{ }^{19-22}$ However, theoretical treatments of the predissociation of each $\mathrm{H}_{3}$ Rydberg state are rather limited, ${ }^{23-25}$ despite of those activities of experimental measurements. Schneider and $\mathrm{Orel}^{25}$ carried out quantum dynamics calculations to estimate the lifetimes of $2 s A_{1}^{\prime}, 3 s A_{1}^{\prime}$, and $3 p E^{\prime}$ states, based on $a b$ initio calculations of the nonadiabatic coupling elements. However, they restricted the nuclear motion of $\mathrm{H}_{3}$ to the $C_{2 v}$ arrangement, to two dimensions. Their study was based on the direct couplings between $2 p E^{\prime}$ and excited 


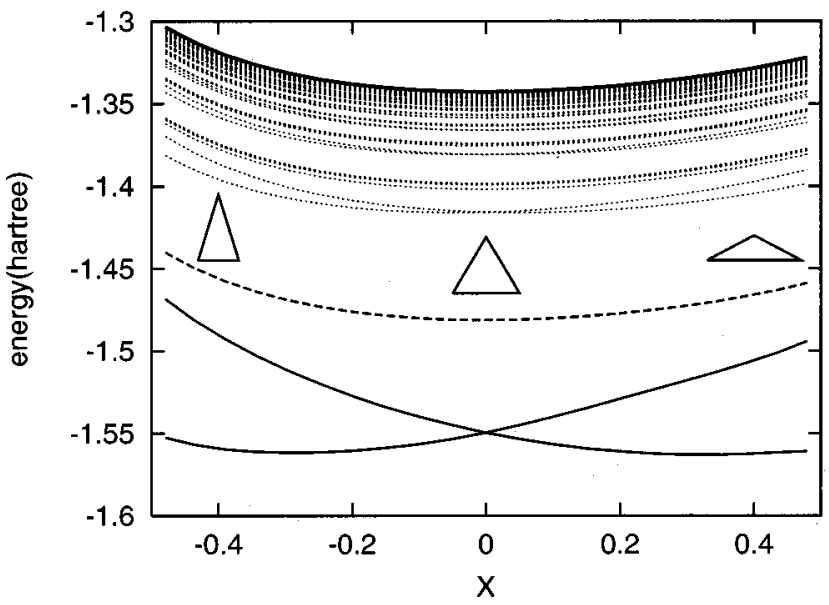

FIG. 1. One-dimensional cut of potential energy surfaces. The upper solid line is the curve for $\mathrm{H}_{3}^{+}$. The dotted lines below $\mathrm{H}_{3}^{+}$indicate Rydberg states. The dashed line represents the $\mathrm{H}_{3} 2 s A_{1}^{\prime}$ state. The two lowest solid lines show the $2 p E^{\prime}$ dissociative state. The upper $2 p E^{\prime}$ state correlates to $\mathrm{H}$ $+\mathrm{H}+\mathrm{H}$, whereas the lower $2 p E^{\prime}$ state correlates to $\mathrm{H}_{2}+\mathrm{H}$. The coordinate $\mathrm{X}$ is defined by the hyperspherical coordinates as $\sin \theta / 2$, which represents the $C_{2 v}$ distortion of the nuclear geometry. Note that these curves were obtained by the one-electron Hamiltonian described later in Sec. II B 1, in combination with the $\mathrm{H}_{3}^{+}$potential surface of Jaquet et al. (Ref. 26).

Rydberg states. The role of the indirect mechanism remains unknown.

In the present paper, we investigate the role of indirect process in the predissociation of $\mathrm{H}_{3}$ Rydberg states. As seen in Fig. 1, the potential energy of the $2 s A_{1}^{\prime}$ state is well separated from a dense higher Rydberg manifold $(n \geqslant 3)$ and the direct predissociation rates from the $3 s A_{1}^{\prime}$ and $3 p E^{\prime}$ states to the dissociative states are considerably smaller compared to that from the $2 s A_{1}^{\prime}$ state as shown by Schneider and Orel. ${ }^{25}$ We therefore paid our attention to the vibronic coupling among higher Rydberg states and their couplings to the vibrationally excited $2 s A_{1}^{\prime}$ states which have large predissociation rates. To realize this picture, we constructed the effective Hamiltonian for describing the dynamics of bound Rydberg states, taking into account the direct nonadiabatic transition effect from the $2 s A_{1}^{\prime}$ to $2 p E^{\prime}$ states. An effective potential for a Rydberg electron was developed to describe the electronic structures of bound Rydberg states. The dynamics is then treated as the combination of the $\mathrm{H}_{3}^{+}$core vibrations and the motion of the Rydberg electron. Note that the vibronic interaction between a Rydberg electron and the $\mathrm{H}_{3}^{+}$ion core is explicitly treated without assuming the BornOppenheimer separation, though two electrons of the $\mathrm{H}_{3}^{+}$ core are assumed to follow the nuclear motion adiabatically.

The organization of this paper is as follows: Sec. II describes theoretical methods both for ab initio electronic structure and quantum dynamics calculations. We construct the effective Hamiltonian for calculating the lifetimes of the Rydberg states. Details of the effective potential for the Rydberg electron are also given. In Sec. III the results of calculations for the energy levels of Rydberg states and their predissociation rates are shown. Comparison with the available experiments is made. We further give a brief discussion about the implication of the present results to the DR process of the $\mathrm{H}_{3}^{+}$ion. The concluding remarks of this paper are summarized in Sec. IV.

\section{THEORETICAL METHOD}

\section{A. Effective Hamiltonian}

In order to treat the predissociation dynamics of $\mathrm{H}_{3}$ Rydberg states, we introduced the effective Hamiltonian in the form,

$$
\hat{H}_{\text {eff }}=\hat{H}_{N}(\mathbf{R})+\hat{H}_{e}(\mathbf{r}, \mathbf{R})+\hat{\Delta}(\mathbf{r}, \mathbf{R})-\frac{i}{2} \hat{\Gamma}(\mathbf{r}, \mathbf{R}),
$$

where $\hat{H}_{N}$ is the the Hamiltonian for the nuclear motion of the $\mathrm{H}_{3}^{+}$ion and $\hat{H}_{e}$ is the one-electron Hamiltonian for the Rydberg electron. The Hermit operators, $\hat{\Delta}$ and $\hat{\Gamma}$, represent the shifts and widths caused by the dissociation. The coordinates of nuclei and Rydberg electron are denoted as $\mathbf{R}$ and $\mathbf{r}$, respectively. In deriving Eq. (1), we employed the Feshbach partitioning technique, ${ }^{27,28}$ where the total molecular vibronic states are divided into $P$-space composed of Rydberg states with bound nuclear vibrations, and $Q$-space corresponding to the valence $2 p E^{\prime}$ electronic states with dissociative continua of nuclear motion. Although this effective Hamiltonian formally depends on the energy, we replaced it with the energy eigenvalue of $\hat{H}_{P P}$ and neglected its energy dependence. This approximation is justified because the offdiagonal elements of $\hat{\Delta}$ and $\hat{\Gamma}$ are usually small compared to the diagonal elements when the effective Hamiltonian is represented by the eigenfunction of $\hat{H}_{P P}$. The explicit expressions of these matrix elements will be shown in Sec. IIC.

We represent this effective Hamiltonian by the diabatic basis, $\chi_{n}(\mathbf{R}) \psi_{m}\left(\mathbf{r}, \mathbf{R}_{0}\right)$, where $\mathbf{R}_{0}$ stands for the equilibrium nuclear geometry of $\mathrm{H}_{3}^{+}$. The vibrational basis function $\chi_{n}$ is the eigenfunction of $\hat{H}_{N}$,

$$
\hat{H}_{N} \chi_{n}(\mathbf{R})=E_{n}^{(N)} \chi_{n}(\mathbf{R}),
$$

and the electronic basis $\psi_{m}$ is the eigenfunction of $\hat{H}_{e}$,

$$
\hat{H}_{e}\left(\mathbf{r}, \mathbf{R}_{0}\right) \psi_{m}\left(\mathbf{r}, \mathbf{R}_{0}\right)=E_{m}^{(e)}\left(\mathbf{R}_{0}\right) \psi_{m}\left(\mathbf{r}, \mathbf{R}_{0}\right) .
$$

Here the $1 s A_{1}^{\prime}$ and $2 p E^{\prime}$ electronic wave functions are omitted from the basis functions. The Hamiltonian $\hat{H}_{\text {eff }}$ is then represented in terms of the basis set $\left|\psi_{m} \chi_{n}\right\rangle$ as

$$
\begin{aligned}
\left\langle\chi_{n^{\prime}} \psi_{m^{\prime}}\left|\hat{H}_{\mathrm{eff}}\right| \chi_{n} \psi_{m}\right\rangle & \\
= & \left(E_{n}^{(N)}+E_{m}^{(e)}\right) \delta_{n^{\prime} n} \delta_{m^{\prime} m}+\left\langle\chi_{n^{\prime}} \psi_{m^{\prime}}\left|\Delta \hat{V}_{e}\right| \chi_{n} \psi_{m}\right\rangle \\
& +\left\langle\chi_{n^{\prime}} \psi_{m^{\prime}}|\hat{\Delta}| \chi_{n} \psi_{m}\right\rangle-\frac{i}{2}\left\langle\chi_{n^{\prime}} \psi_{m^{\prime}}|\hat{\Gamma}| \chi_{n} \psi_{m}\right\rangle
\end{aligned}
$$

and

$$
\Delta \hat{V}_{e}=\hat{V}_{e}(\mathbf{r}, \mathbf{R})-\hat{V}_{e}\left(\mathbf{r}, \mathbf{R}_{0}\right),
$$

where $\hat{V}_{e}$ is the potential term of the one-electron Hamiltonian $\hat{H}_{e}$ in Eq. (1). The operators $\widehat{\Delta}$ and $\hat{\Gamma}$ are represented as 


$$
\begin{aligned}
& \left\langle\chi_{n^{\prime}} \psi_{m^{\prime}}|\hat{\Delta}| \chi_{n} \psi_{m}\right\rangle \\
& \quad=\sum_{i}\left\langle\chi_{n^{\prime}} \psi_{m^{\prime}} \mid \chi_{i}^{(2 s)} \psi^{(2 s)}\right\rangle \Delta_{i}^{(2 s)}\left\langle\chi_{i}^{(2 s)} \psi^{(2 s)} \mid \chi_{n} \psi_{m}\right\rangle,
\end{aligned}
$$

and

$$
\begin{aligned}
& \left\langle\chi_{n^{\prime}} \psi_{m^{\prime}}|\hat{\Gamma}| \chi_{n} \psi_{m}\right\rangle \\
& \quad=\sum_{i}\left\langle\chi_{n^{\prime}} \psi_{m^{\prime}} \mid \chi_{i}^{(2 s)} \psi^{(2 s)}\right\rangle \Gamma_{i}^{(2 s)}\left\langle\chi_{i}^{(2 s)} \psi^{(2 s)} \mid \chi_{n} \psi_{m}\right\rangle .
\end{aligned}
$$

The matrix element $\left\langle\chi_{n^{\prime}} \psi_{m^{\prime}} \mid \chi_{i}^{(2 s)} \psi^{(2 s)}\right\rangle$ is the overlap integral between the diabatic basis $\chi_{n^{\prime}}(\mathbf{R}) \psi_{m^{\prime}}\left(\mathbf{r}, \mathbf{R}_{0}\right)$ and the adiabatic basis $\chi_{i}^{(2 s)}(\mathbf{R}) \psi^{(2 s)}(\mathbf{r}, \mathbf{R})$, where $\psi^{(2 s)}$ is the electronic wave function of $2 s$ state and $\chi_{i}^{(2 s)}$ is the $i$ th vibrational wave function on the $2 s$ surface. We renormalized these matrix elements so as to satisfy the condition,

$$
\sum_{n m}\left|\left\langle\chi_{n} \psi_{m} \mid \chi_{i}^{(2 s)} \psi^{(2 s)}\right\rangle\right|^{2}=1
$$

which is intended to correctly reproduce the $2 s$ decay widths obtained by $a b$ initio calculations. We used the diagonal widths $\Gamma_{i}^{(2 s)}$ and shifts $\Delta_{i}^{(2 s)}$ of $2 s$ state in Eq. (7) and neglected the off-diagonal terms, because they were typically 3-5 orders of magnitude smaller compared to the diagonal terms.

At this point, the relation between the coordinates $\mathbf{r}$ and $\mathbf{R}$ is not specified. We placed the $i$ th proton in $\mathbf{r}$-space according to the mapping $x_{i}(\mathbf{R}), y_{i}(\mathbf{R})$ and $z_{i}(\mathbf{R})$ which satisfies the Eckart condition, ${ }^{29}$

$\sum_{i=1,3} m_{i} x_{i}(\mathbf{R})=\sum_{i=1,3} m_{i} y_{i}(\mathbf{R})=0, \quad z_{i}(\mathbf{R})=0 \quad(i=1,2,3)$

and

$$
\sum_{i=1,3} m_{i}\left\{x_{i}\left(\mathbf{R}_{0}\right) \Delta y_{i}(\mathbf{R})-y_{i}\left(\mathbf{R}_{0}\right) \Delta x_{i}(\mathbf{R})\right\}=0
$$

with

$$
\Delta x_{i}(\mathbf{R})=x_{i}(\mathbf{R})-x_{i}\left(\mathbf{R}_{0}\right), \quad \Delta y_{i}(\mathbf{R})=y_{i}(\mathbf{R})-y_{i}\left(\mathbf{R}_{0}\right) .
$$

The first condition places the center-of-mass of the $\mathrm{H}_{3}^{+}$ion at the origin of the r-space with the molecular plane being the $x y$-plane. The second condition determines the rotation angle around the $z$-axis. Under these conditions, the integral $\left\langle\chi_{n^{\prime}} \psi_{m^{\prime}}\left|\Delta \hat{V}_{e}\right| \chi_{n} \psi_{m}\right\rangle$ preserves the $D_{3 h}$ symmetry of the system.

\section{B. Electronic and vibrational basis for Rydberg states}

\section{Electronic basis}

The potential term in the one-electron Hamiltonian $\hat{H}_{e}(\mathbf{r}, \mathbf{R})$ is approximated as the sum of the Coulomb potential of the $\mathrm{H}_{3}^{+}$ion core and the exchange one. The Coulomb potential is represented as

$$
V_{\mathrm{cl}}(\mathbf{r}, \mathbf{R})=-\sum_{i=1,3} \frac{Z_{i}^{\mathrm{eff}}(\mathbf{r}, \mathbf{R})}{\left|\mathbf{R}_{i}-\mathbf{r}\right|} .
$$

Here the effective charge on each proton, $Z_{i}^{\text {eff }}(\mathbf{r}, \mathbf{R})$, includes the shielding effect by two electrons occupying the $1 a_{1}^{\prime}$ orbital of $\mathrm{H}_{3}^{+}$. The charge $Z_{i}^{\text {eff }}$ should approach to unity near the proton position $\mathbf{R}_{i}$ and have the value $Z_{i}$ away from the proton, where $Z_{i}$ is the partial charge obtained to reproduce the $\mathrm{H}_{3}^{+}$electrostatic potential at the outer region. We used the exponential function to represent this shielding effect as

$$
Z_{i}^{\text {eff }}(\mathbf{r}, \mathbf{R})=Z_{i}(\mathbf{R})+\left\{1-Z_{i}(\mathbf{R})\right\} \exp \left(-\gamma\left|\mathbf{R}_{i}-\mathbf{r}\right|\right) .
$$

Although the exchange effect is formally represented by a nonlocal potential, we employed the local approximation based on free-electron gas model ${ }^{30}$ because of its simplicity. We will show in Figs. 4 and 5 that the present model can reproduce the $a b$ initio Rydberg energies reasonably well. In this paper, we used the following form of the exchange potential $^{31}$ which have been used in the literature, ${ }^{32,33}$

$$
V_{\mathrm{ex}}(\mathbf{r}, \mathbf{R})=-A(\mathbf{R}) \frac{2}{\pi} k_{F} F(\eta)
$$

where

$$
\begin{aligned}
& k_{F}(\mathbf{r}, \mathbf{R})=\left\{3 \pi^{2} \varrho(\mathbf{r}, \mathbf{R})\right\}^{1 / 3}, \\
& F(\eta)=\frac{1}{2}+\frac{1-\eta^{2}}{4 \eta} \ln \left|\frac{1+\eta}{1-\eta}\right|,
\end{aligned}
$$

and

$$
\eta(\mathbf{r}, \mathbf{R})=\left(k^{2}+2 I+k_{F}^{2}\right)^{1 / 2} / k_{F} .
$$

Here $\varrho(\mathbf{r}, \mathbf{R})$ is the density of $H_{3}^{+} 1 a_{1}^{\prime}$ electrons. The wave number $k$ of the incident electron is set to zero in this work. In these equations, $I$ represents the ionization potential and $A(\mathbf{R})$ is the multiplication factor depending on the nuclear geometry. The ionization potential was chosen to be 1.2 hartree. We will discuss the explicit functional form of $A(\mathbf{R})$ in Sec. III A.

In order to represent Rydberg orbitals, we need to use a basis set that resolves a region around the ion core and also covers a large distance region from the core. This condition is needed because the changes of Rydberg orbitals near $\mathrm{H}_{3}^{+}$ play an important role for the nonadiabatic transition among Rydberg states, although they extend far away from the core region. To achieve this requirement, we used the mapping procedure proposed by Fattal et al., ${ }^{34}$ in which the radial coordinate $r$ is transformed to the new variable $Q$ as

$$
r=Q-\alpha \arctan (\beta Q)
$$

where $\alpha$ and $\beta$ are the control parameters for the mapping. The radial part of the electron kinetic energy operator is written as

$$
\hat{T}_{e}^{\mathrm{rad}}=-\frac{\hbar^{2}}{2 m_{e}}\left(J^{-1 / 2} \frac{\partial}{\partial Q} J^{-1 / 2}\right)^{2},
$$

where $J$ is the Jacobian of the transformation,

$$
J(Q)=\frac{d r}{d Q} .
$$

The angular part is the same as usual, 
$\hat{T}_{e}^{\text {(ang) }}=-\frac{\hbar^{2}}{2 m_{e} r^{2}}\left[\frac{1}{\sin \theta_{e}} \frac{\partial}{\partial \theta_{e}}\left(\sin \theta_{e} \frac{\partial}{\partial \theta_{e}}\right)+\frac{1}{\sin ^{2} \theta_{e}} \frac{\partial^{2}}{\partial \phi_{e}^{2}}\right]$.

We used the discrete variable representation (DVR) for the $Q$ variable and the DVR points were obtained by diagonalizing the matrix $\left\langle f_{n}|Q| f_{m}\right\rangle$, where $f_{n}$ is the sine function,

$$
f_{n}(Q)=\sqrt{2 / Q_{0}} \sin \left(n \pi Q / Q_{0}\right),
$$

with $n$ being integer. The spherical harmonics $Y_{l m}\left(\theta_{e}, \phi_{e}\right)$ were used to represent the angular part.

In the actual calculations, we took $Q_{0}=1600$ bohr, $\alpha$ $=1599.0$, and $\beta=0.000625$. The value of $r$ corresponding $Q_{0}$ is 343 bohr in this case. With these parameters, Rydberg energies were well reproduced up to the principal quantum number $n=11$. For the case of the hydrogen atom, the error of the calculated energy to the exact one, $\mid\left(E_{\text {calc }}\right.$ $\left.-E_{\text {exact }}\right) / E_{\text {exact }} \mid$, is $10^{-5}$ for $n=11$ and $10^{-3}$ for $n=12$. The same accuracy was achieved for $\mathrm{H}_{3}$ Rydberg energies.

\section{Vibrational basis}

We employed the hyperspherical coordinates $\rho, \theta$, and $\phi$ used by Varandas and $\mathrm{Yu}^{35}$ to represent the nuclear geometry. The rotationless nuclear Hamiltonian $\hat{H}_{N}$ in Eq. (1) has the form,

$$
\begin{aligned}
\hat{H}_{N}= & -\frac{\hbar^{2}}{2 \mu}\left\{\frac{\partial^{2}}{\partial \rho^{2}}+\frac{16}{\rho^{2}}\left[\frac{1}{\sin \theta} \frac{\partial}{\partial \theta} \sin \theta \frac{\partial}{\partial \theta}\right.\right. \\
& \left.\left.+\frac{1}{4 \sin ^{2}(\theta / 2)} \frac{\partial^{2}}{\partial \phi^{2}}\right]\right\}+\frac{15 \hbar^{2}}{8 \mu \rho^{2}}+W(\rho, \theta, \phi),
\end{aligned}
$$

where $\mu$ is the reduced mass and $W$ is the potential function of the $\mathrm{H}_{3}^{+}$taken from Jaquet et al. ${ }^{26}$

The vibrational wave functions $\chi_{i}$ were prepared by diagonalization of Eq. (23). The DVR basis of Colbert and Miller $^{36}$ was used for the hyperradius $\rho$. The DVR points were placed in the range $1.3 \leqslant \rho \leqslant 5.3 \mathrm{bohr}$ with the spacing of 0.1 bohr. For the hyperangles $\theta$ and $\phi$, we used the hyperspherical harmonics, ${ }^{37}$ which are the eigenfunctions of the angular part of the nuclear Hamiltonian Eq. (23). Details of the procedure are given in our previous work. ${ }^{38}$

\section{Width and shift matrices of $2 s A_{1}^{\prime}$ state}

The time-dependent version of Fermi's Golden Rule was used to obtain the predissociation widths and shifts of the $2 s$ state as follows:

$$
\Gamma_{i}^{(2 s)}=\frac{2}{\hbar} \operatorname{Re} \int_{0}^{\infty} d t \exp \left(\frac{i E_{i} t}{\hbar}\right)\langle\phi(0) \mid \phi(t)\rangle,
$$

and

$$
\Delta_{i}^{(2 s)}=\frac{1}{\hbar} \operatorname{Im} \int_{0}^{\infty} d t \exp \left(\frac{i E_{i} t}{\hbar}\right)\langle\phi(0) \mid \phi(t)\rangle
$$

with

$$
|\phi(t)\rangle=\exp \left(-\frac{i \hat{H} t}{\hbar}\right) \hat{V}\left|\chi_{i}\right\rangle
$$

where $\chi_{i}$ is the vibrational wave function of the $\mathrm{H}_{3} 2 s A_{1}^{\prime}$ adiabatic electronic state and $E_{i}$ is its energy. The operator $\hat{V}$ in Eq. (26) represents the nonadiabatic coupling,

$$
\begin{aligned}
\hat{V}= & -\frac{\hbar^{2}}{2 \mu}\left[2\left\langle\psi_{d}\left|\frac{\partial}{\partial \rho}\right| \psi_{2 s}\right\rangle \frac{\partial}{\partial \rho}+\frac{32}{\rho^{2}}\left\langle\psi_{d}\left|\frac{\partial}{\partial \theta}\right| \psi_{2 s}\right\rangle \frac{\partial}{\partial \theta}\right. \\
& +\frac{16 \cos \theta}{\rho^{2} \sin \theta}\left\langle\psi_{d}\left|\frac{\partial}{\partial \theta}\right| \psi_{2 s}\right\rangle \\
& \left.+\frac{8}{\rho^{2} \sin ^{2}(\theta / 2)}\left\langle\psi_{d}\left|\frac{\partial}{\partial \phi}\right| \psi_{2 s}\right\rangle \frac{\partial}{\partial \phi}\right] .
\end{aligned}
$$

Here $\left\langle\psi_{d}|\partial / \partial \rho| \psi_{2 s}\right\rangle,\left\langle\psi_{d}|\partial / \partial \theta| \psi_{2 s}\right\rangle$, and $\left\langle\psi_{d}|\partial / \partial \phi| \psi_{2 s}\right\rangle$ are the nonadiabatic coupling matrix elements between the dissociative $2 p E^{\prime}$ electronic state $\psi_{d}$ and $2 s A_{1}^{\prime}$ Rydberg state $\psi_{2 s}$. Note that Eqs. (24) and (25) represent the diagonal matrix elements of width and shift operators. For the offdiagonal matrix elements of width operator, we used the following equation:

$$
\Gamma_{i j}^{(2 s)}=\frac{2}{\hbar} \operatorname{Re} \int_{0}^{\infty} d t \exp \left(\frac{i E t}{\hbar}\right)\left\langle\chi_{i}\left|\hat{V}^{\dagger} \exp \left(-\frac{i \hat{H} t}{\hbar}\right) \hat{V}\right| \chi_{j}\right\rangle,
$$

where $E$ is the average of $E_{i}$ and $E_{j}$. The off-diagonal elements of shift operator have the similar form as well.

We carried out $a b$ initio calculations to obtain the nonadiabatic coupling elements using MOLPRO98 package. ${ }^{39}$ The state-averaged complete active space self-consistent field (CASSCF) method man $^{40,4}$ was employed. We placed $(9 s 4 p) /[6 s 4 p]$ basis set at each position of proton and $(5 s 5 p 2 d)$ basis at the center-of-mass. The exponents and contraction coefficients were taken from Schneider and Orel. ${ }^{25}$ The analytic gradient method was used to calculate the nonadiabatic coupling elements.

The initial vibrational wave functions $\chi_{i}$ were prepared by diagonalizing Eq. (23), where the potential term $W$ was replaced by the ab initio $\mathrm{H}_{3} 2 s$ surface. The wave packet $\hat{V}\left|\chi_{i}\right\rangle$ was propagated by the Chebychev polynomial method $^{42}$ on the $2 p E^{\prime}$ surfaces. ${ }^{43}$ We used the same angular basis as for the $H_{3}^{+}$vibrations. For the hyperradius $\rho$, the grid points were extended up to 9.3 bohr.

We calculated the diagonal and off-diagonal elements for the width and shift matrices. Since the off-diagonal terms were 3-5 orders of magnitude smaller compared to the diagonal ones, we used only the diagonal terms for constructing the effective Hamiltonian.

\section{RESULTS AND DISCUSSION}

\section{A. Rydberg electronic states}

We diagonalized the one-electron Hamiltonian, $\hat{H}_{e}(\mathbf{r}, \mathbf{R})$, to obtain the electronic energy levels of Rydberg states. The partial charge $Z_{i}(\mathbf{R})$ in Eq. (13) was obtained by Distributed Multipole Analysis ${ }^{44}$ and the parameter $\gamma$ was chosen to be 2.0 so as to reproduce the $a b$ initio electrostatic potential as shown in Fig. 2. The electronic density $\varrho(\mathbf{r}, \mathbf{R})$ required to construct the local exchange potential, Eq. (14), was obtained by $a b$ initio calculations. The multiplication factor $A(\mathbf{R})$ in Eq. (14) was determined to reproduce the $a b$ initio 

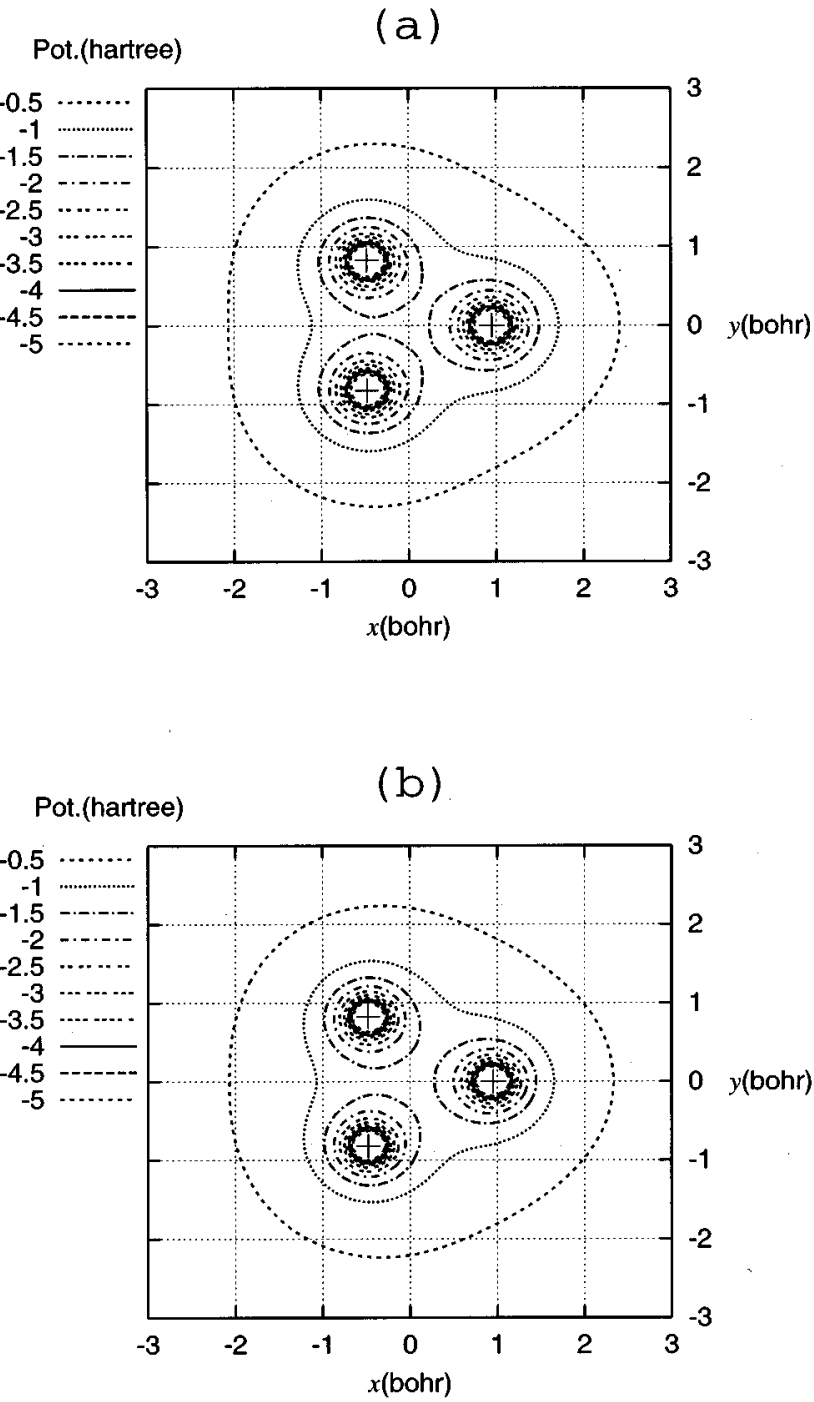

FIG. 2. Electrostatic potential around $\mathrm{H}_{3}^{+}$produced by (a) ab initio calculation and (b) Eq. (12). The $z=0$ cut of the $x y$-plane is shown. The three protons are located on the same plane, as indicated by the cross marks.

energies of $\mathrm{H}_{3}$ Rydberg states, which were calculated by the state-averaged CASSCF method with the same basis set for the calculations of nonadiabatic coupling elements. The resultant form of $A(\mathbf{R})$ was given as

$$
A(\rho, \theta, \phi)=c_{0}+c_{1} \rho,
$$

where the parameters $c_{0}$ and $c_{1}$ were chosen to be -0.95 and 1.60 , respectively. Here the hyperradius $\rho$ is given in bohr unit. In Fig. 3, the exchange potential $V_{\text {ex }}$ is compared with the Coulomb potential $V_{\mathrm{cl}}$ and the one-electron potential $V_{e}$. As shown in the figure, the one-electron potential is dominated by the Coulomb term and the contribution of the exchange term is noticeable only around the center. This behavior explains the trend that the exchange potential mainly affects the energy of $s$ and $p$ Rydberg states.

The Rydberg state energies obtained from the oneelectron Hamiltonian are compared with those from ab initio state-averaged CASSCF calculations in Figs. 4 and 5. In

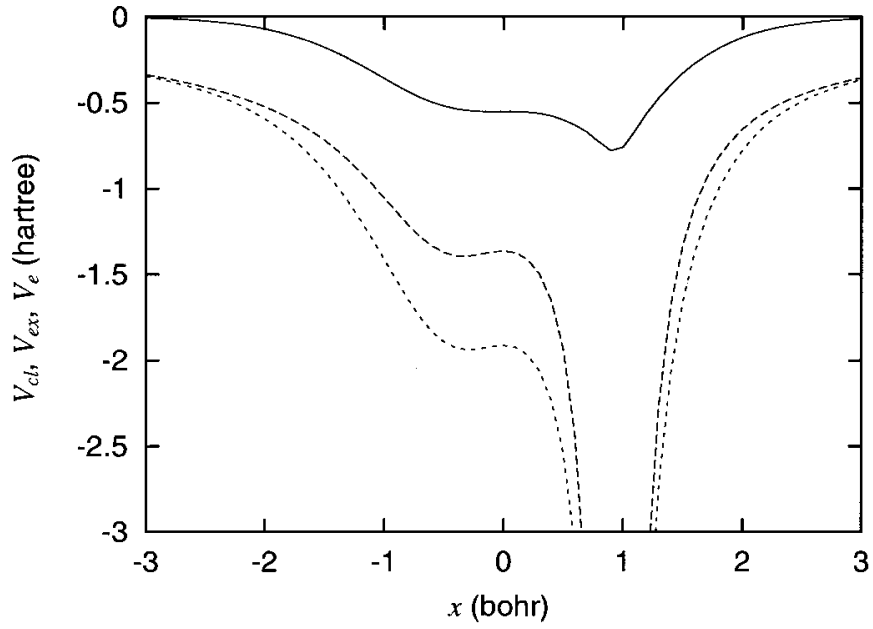

FIG. 3. One-electron potentials $V_{e}, V_{\mathrm{cl}}, V_{\mathrm{ex}}$, through line $y=0$ and $z$ $=0$. The positions of the protons are the same as Fig. 2. The solid line is $V_{\mathrm{ex}}$, the dashed line is $V_{\mathrm{cl}}$ and the dotted line is $V_{e}$.

these figures, the energy is represented by the effective quantum number $n_{\text {eff }}$ defined as

$$
E\left(\mathrm{H}_{3}\right)-E\left(\mathrm{H}_{3}^{+}\right)=-\frac{0.5}{n_{\mathrm{eff}}^{2}} \text { (hartree) } .
$$

Figure 4 shows the dependence of the energies on the $C_{2 v}$ distortion of the $\mathrm{H}_{3}$ molecule. The $\mathrm{H}_{3}$ energies at the equilibrium geometry of $\mathrm{H}_{3}^{+}$are well reproduced and the JahnTeller energy splittings of $p E^{\prime}$ states also agree. Figure 5 shows the dependence of the energies on the $D_{3 h}$ geometry distortion. These energies well reproduce the ab initio ones. It is noted that the nuclear geometry dependence of $A(\mathbf{R})$ in Eq. (29) is intended to reproduce the $\rho$ dependence of $p E^{\prime}$ quantum defects. The $\rho$ independent multiplication factor $A$ provided rather constant $p E^{\prime}$ quantum defects with respect to the $D_{3 h}$ distortion, although $s A_{1}^{\prime}$ states still agreed well with the $a b$ initio results.

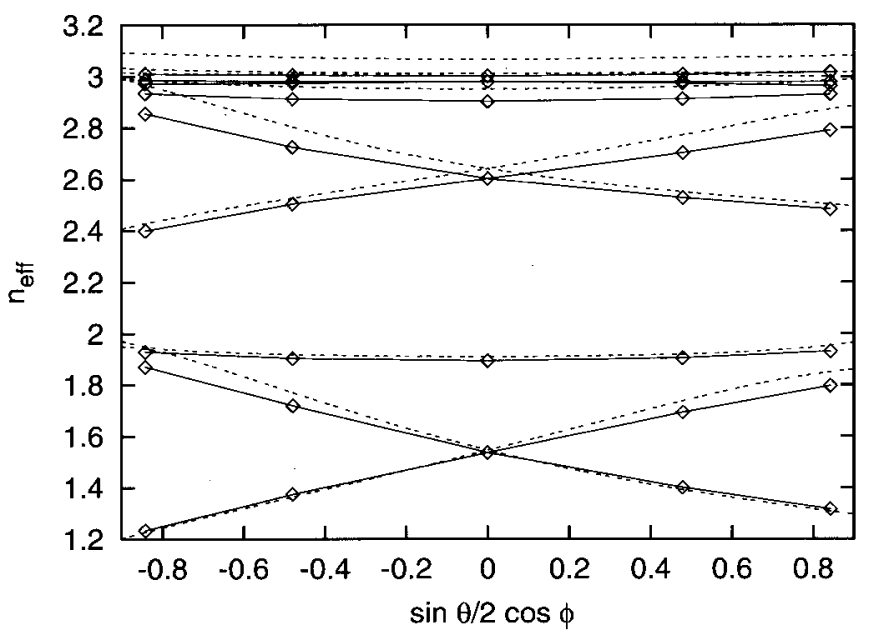

FIG. 4. Effective quantum numbers along the $C_{2 v}$ distortion. The solid lines are obtained by the one-electron potential and the dotted lines are by the $a b$ initio calculation. The hyperradius is fixed to $\rho=2.17 \mathrm{bohr}$. The value of $\phi$ is 0 for $\sin \theta / 2 \cos \phi \geqslant 0$ and $\pi$ for $\sin \theta / 2 \cos \phi \leqslant 0$. The equilibrium geometry of $\mathrm{H}_{3}^{+}$corresponds $\theta=0$. 


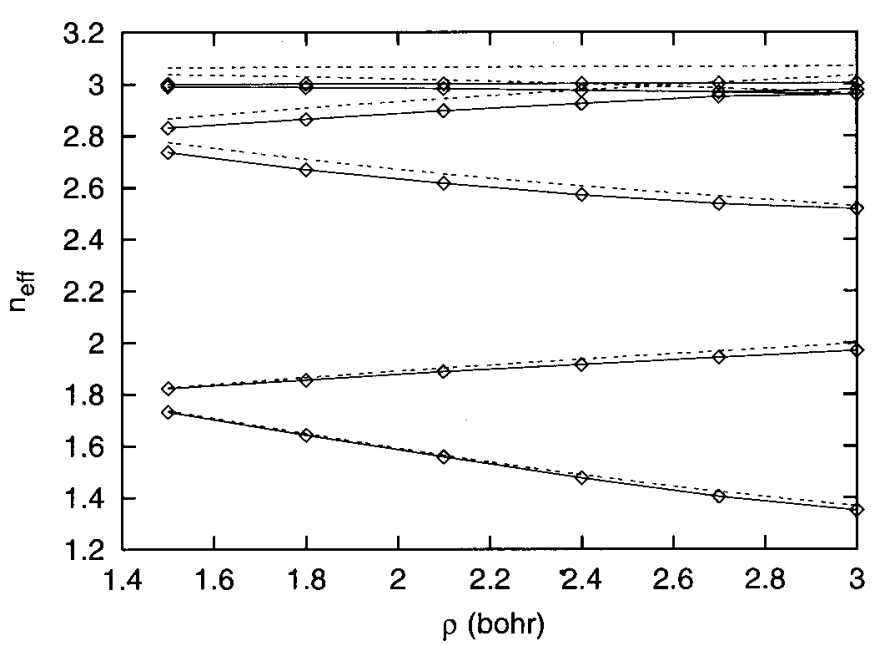

FIG. 5. Effective quantum numbers along $D_{3 h}$ distortion. The solid lines are obtained by an one-electron potential and the dotted lines are by $a b$ initio calculation. The hyperangle $\theta$ is fixed to 0 .

\section{B. Predissociation widths and shifts of the $2 s$ state}

We calculated the nonadiabatic coupling elements between the $2 s A_{1}^{\prime}$ Rydberg and the $2 p E^{\prime}$ dissociative electronic states as functions of the hyperspherical coordinates $\rho$, $\theta$, and $\phi$. The representative results are shown in Fig. 6, where we can see that the couplings to the upper dissociative surface are generally larger than those to the lower surface. It is also seen that the couplings are larger at small $\rho$. From these results, the $2 s$ state is expected to decay into the upper $2 p$ surface predominantly passing through compact geometry of $\mathrm{H}_{3}$.

The predissociation widths and shifts were obtained for 600 vibrational states of the $2 s$ electronic state. The resultant decay widths are shown in Fig. 7. For the ground vibrational level, the calculated rate is $2.0 \times 10^{13} \mathrm{~s}^{-1}$ which is close to the experimental value ${ }^{15}$ of about $6 \times 10^{12} \mathrm{~s}^{-1}$. The widths were averaged over 70 states at each energy window and the (a)

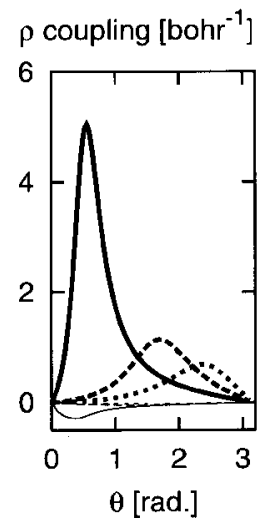

(b)

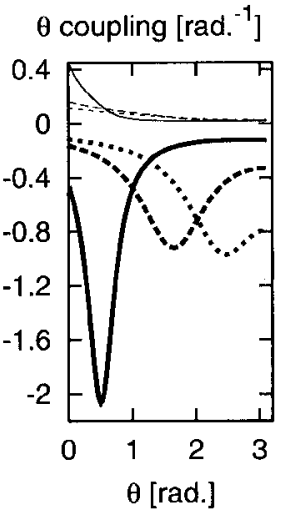

(c)

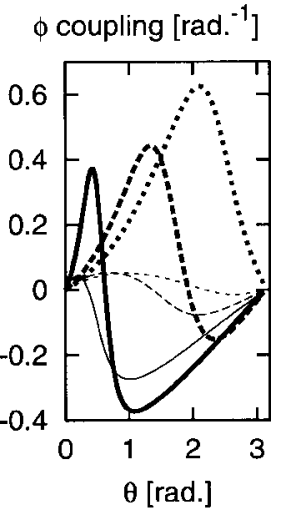

FIG. 6. Nonadiabatic coupling elements of $2 s A_{1}^{\prime}$ with $2 p E^{\prime}$ dissociative states at the hyperangle $\phi=\pi / 6$. Each panel corresponds to the coupling as (a) $\left\langle\psi_{d}|\partial / \partial \rho| \psi_{2 s}\right\rangle$, (b) $\left\langle\psi_{d}|\partial / \partial \theta| \psi_{2 s}\right\rangle$, and (c) $\left\langle\psi_{d}|\partial / \partial \phi| \psi_{2 s}\right\rangle$. The thick lines are the couplings between $2 s$ and upper $2 p E^{\prime}$ state, and the thin lines represent the couplings between $2 s$ and lower $2 p E^{\prime}$ state. Solid lines are for $\rho=1.5 \mathrm{bohr}$, dashed lines for $\rho=2.1 \mathrm{bohr}$, and dotted lines for $\rho$ $=2.7 \mathrm{bohr}$.

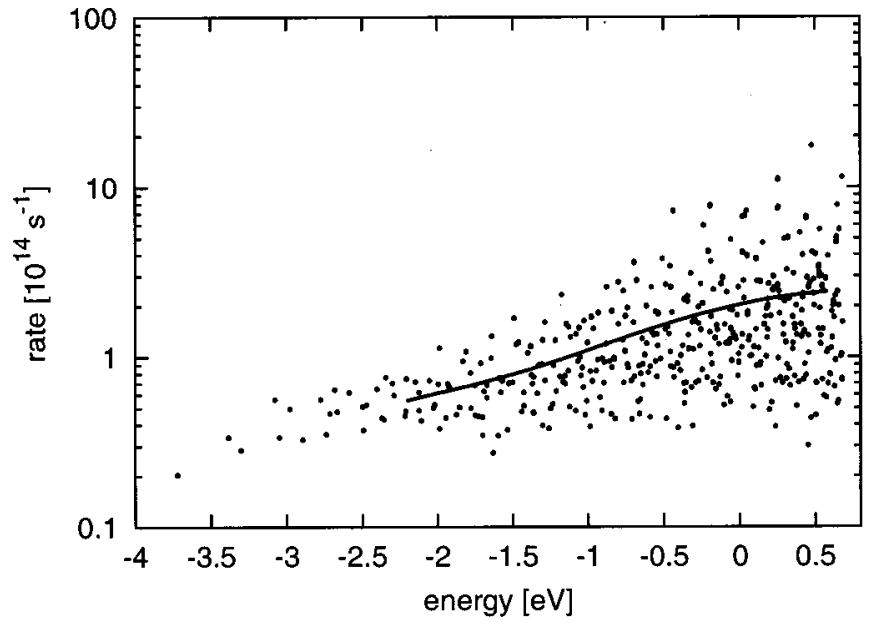

FIG. 7. Predissociation rates $\Gamma_{i} / \hbar$ of the vibrationally excited $\mathrm{H}_{3} 2 s$ states. The energy of each state is given with respect to the ground vibrational level of $\mathrm{H}_{3}^{+}$. The solid line represents average rate.

result is also included in Fig. 7. The average width increases with increasing the vibrational energy, and becomes about $2.0 \times 10^{14} \mathrm{~s}^{-1}$ at $E=0 \mathrm{eV}$, which corresponds to the energy of the $\mathrm{H}_{3}^{+}$vibrational ground. Note that the fluctuation around the average width is large even at a high energy region. The energy shifts are presented in Fig. 8, where the shift is $1.4 \mathrm{~cm}^{-1}$ for the ground vibrational state. As the vibrational energy increases, the shift becomes large with a significant scatter.

\section{Predissociation of higher Rydberg states}

\section{Complex eigenvalues of the effective Hamiltonian}

The effective Hamiltonian $\hat{H}_{\text {eff }}$ was diagonalized to obtain the complex eigenvalues $\widetilde{E}_{i}-i \widetilde{\Gamma}_{i} / 2$. To construct the diabatic basis, the electronic bases $\psi_{n}$ were prepared for the Rydberg states with the principal quantum number $n$ $=2-10$ with the angular momentum $l \leqslant 3$. As already noted,

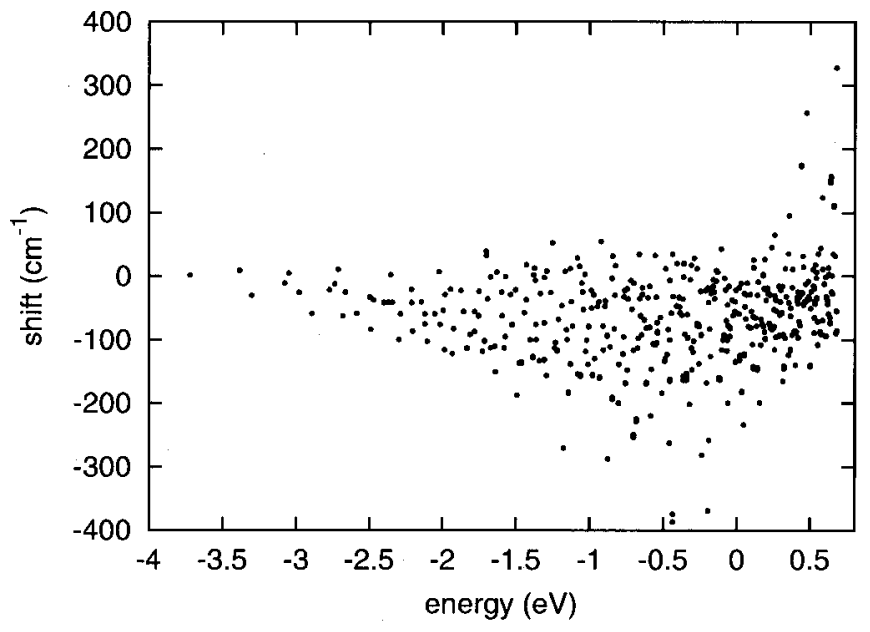

FIG. 8. Energy shifts $\Delta_{i}$ of $2 s$ vibrational states. The origin of the energy is the same as Fig. 7. 


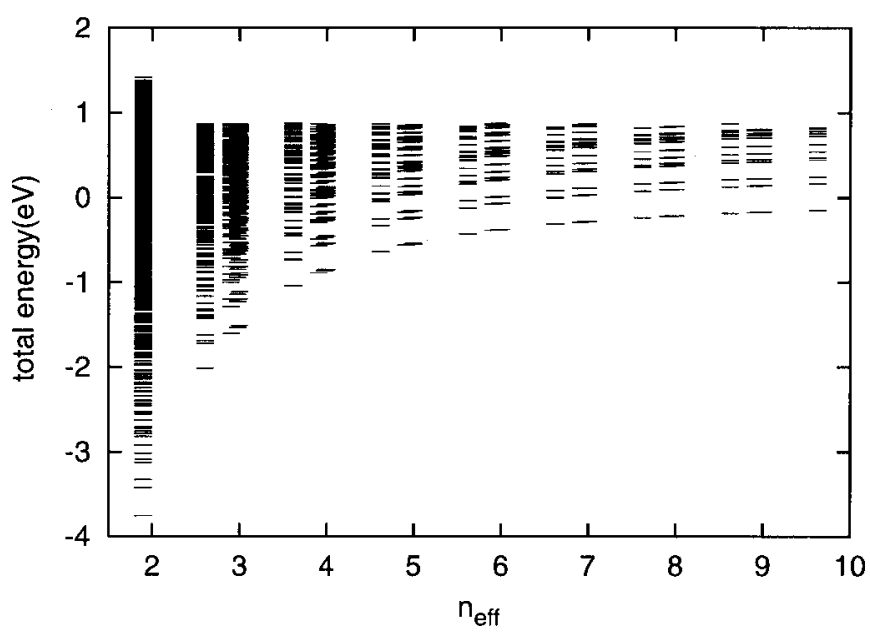

FIG. 9. Energy levels of diabatic states. For each diabatic base $\chi_{n} \psi_{m}$, the total energy is plotted against the effective quantum number of the electronic base $\psi_{m}$. The origin of the total energy is the same as Fig. 7.

the $2 p E^{\prime}$ states were not included in this set. We further added one positive energy state with $A_{1}^{\prime}$ symmetry to observe the effect of autoionization. A total of 1000 vibrational bases $\chi_{m}$ were also prepared. The energy of these bases extends up to $4.8 \mathrm{eV}$ from the vibrational ground state. From the products of these electronic and vibrational bases, we selected 4000 diabatic bases $\psi_{n} \chi_{m}$ according to the energy criterion $E \leqslant 0.05$ hartree, except for the $2 s A_{1}^{\prime}$ state. These are shown in Fig. 9 with their energies and effective quantum numbers. Note that we took the diagonal part of the Hamiltonian $\hat{H}_{P P}$ as the energy of the diabatic state.

The complex eigenvalues $\widetilde{E}_{i}-i \widetilde{\Gamma}_{i} / 2$ are shown in Fig. 10 , where the decay widths $\widetilde{\Gamma}_{i}$ are converted to the predissociation rates. In the upper part of the figure, fast decay rates of the vibrationally excited $2 s A_{1}^{\prime}$ states are extended as a horizontal band, which consists of 577 points. The distribution and average value of these rates are almost the same as those shown in Fig. 7, which were derived from the wave packet calculations. In the middle to lower part of the figure,

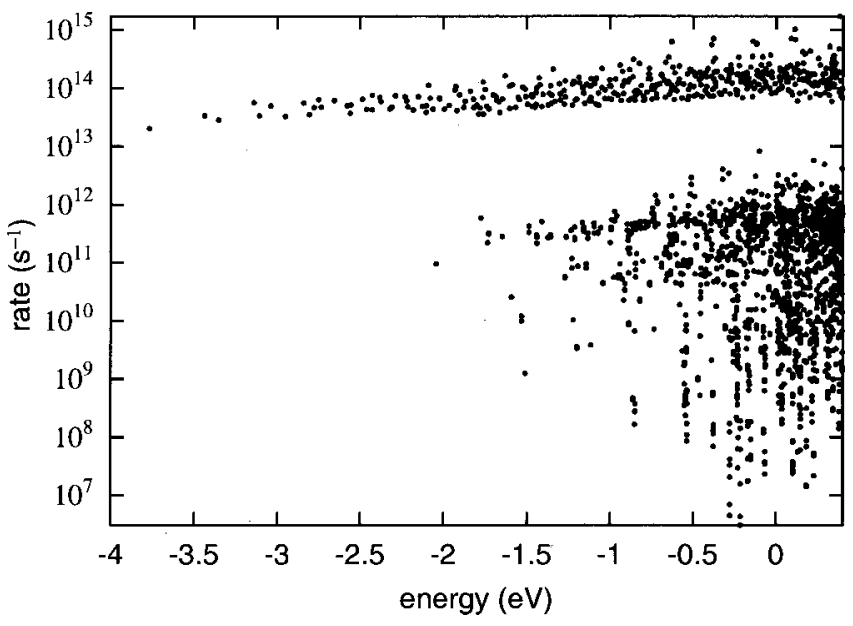

FIG. 10. Energies and decay rates obtained by the effective Hamiltonian. The origin of the energy is the same as Fig. 7.
TABLE I. Comparison of energy differences with experiments.

\begin{tabular}{crr}
\hline \hline State & This work $\left(\mathrm{cm}^{-1}\right)$ & Expt. $\left(\mathrm{cm}^{-1}\right)$ \\
\hline $2 s A_{1}^{\prime}\left\{00^{0}\right\}$ & -17556 & $-17593^{\mathrm{a}}$ \\
$3 s A_{1}^{\prime}\left\{00^{0}\right\}$ & 0 & $0^{\mathrm{b}}$ \\
$3 s A_{1}^{\prime}\left\{10^{0}\right\}$ & 3227 & $3212^{\mathrm{c}}$ \\
$4 s A_{1}^{\prime}\left\{00^{0}\right\}$ & 5680 & $5727^{\mathrm{c}}$ \\
$4 s A_{1}^{\prime}\left\{10^{0}\right\}$ & 8888 & $8936^{\mathrm{d}}$ \\
$5 s A_{1}^{\prime}\left\{00^{0}\right\}$ & 8303 & $8337^{\mathrm{c}}$ \\
$5 s A_{1}^{\prime}\left\{10^{0}\right\}$ & 11500 & $11528^{\mathrm{d}}$ \\
$6 s A_{1}^{\prime}\left\{00^{0}\right\}$ & 9707 & $9740^{\mathrm{d}}$ \\
$6 s A_{1}^{\prime}\left\{10^{0}\right\}$ & 12870 & $12924^{\mathrm{e}}$ \\
$7 s A_{1}^{\prime}\left\{00^{0}\right\}$ & 10553 & $10583^{\mathrm{d}}$ \\
$7 s A_{1}^{\prime}\left\{10^{0}\right\}$ & 13735 & $13762^{\mathrm{e}}$ \\
$3 p E^{\prime}\left\{01^{1}\right\}$ & -1136 & $-1146^{\mathrm{c}}$ \\
$4 p E^{\prime}\left\{01^{1}\right\}$ & 7039 & $7111^{\mathrm{c}}$ \\
\hline \hline
\end{tabular}

${ }^{a}$ Dabowski and Herzberg (Ref. 15).

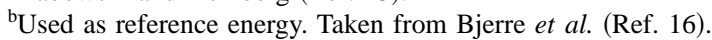

${ }^{c}$ Mistrík et al. (Ref. 8).

${ }^{\mathrm{d}}$ Lembo and Helm (Ref. 45).

${ }^{\mathrm{e}}$ Observed in vibrational autoionization (Ref. 45).

the decay rates of higher Rydberg states are distributed. The points inside this region amount to 1344 . This $n \geqslant 3$ Rydberg component has large vertical extent from the fast decay rate of $10^{12} \mathrm{~s}^{-1}$ to the slow rate of $10^{7} \mathrm{~s}^{-1}$. The number of states with the lifetimes shorter than 1 ps is 82 , whereas the number increases to 708 for the lifetimes shorter than $10 \mathrm{ps}$, indicating that more than half of the higher Rydberg states dissociates within 10 ps. Each eigenstate is usually dominated by a single diabatic state $\chi_{n} \psi_{m}$, except for the states having large widths. For this reason, we sometimes label the eigenstate by its dominating diabatic state hereafter. The eigenstates with fast decay rates, about $10^{12-13} \mathrm{~s}^{-1}$, are mainly composed of the $n=3$ Rydberg states with high vibrational excitations. In contrast, the eigenstates with slow rates, $<10^{10} \mathrm{~s}^{-1}$, are mainly composed of the $d$ and $f$ Rydberg states with the ground vibrational state or singly excited vibrational states. The eigenstates composed of $s$ and $p$ Rydberg states have relatively fast decay rates in many cases, irrespective of their principal quantum numbers and vibrational states, although some states exhibit very slow rates. We classified these eigenstates according to their symmetry, which is given as the direct product of electronic and vibrational symmetries. For the $2 s A_{1}^{\prime}$ electronic states, $A_{1}^{\prime}$ states tend to have slow rates compared to the $A_{2}^{\prime}$ and $E^{\prime}$ states, as already shown in our previous work. ${ }^{38}$ For higher Rydberg states, no significant trend was observed among the $A_{1}^{\prime}, A_{2}^{\prime}$ and $E^{\prime}$ symmetries, however.

We picked up some representative eigenstates to compare with the experiments. The energies of these eigenstates are given in Table I. Although the experimental energies are measured with respect to the $2 p A_{2}^{\prime \prime}(N=0, K=0)\left\{00^{\circ}\right\}$ state, we measured the energies relative to the $3 s A_{1}^{\prime}\left\{00^{\circ}\right\}$ state, since our bases did not include $A_{2}^{\prime \prime}$ and $E^{\prime \prime}$ electronic states. The results agree the experiments within about $100 \mathrm{~cm}^{-1}$.

The decay rates of representative eigenstates are listed in Table II along with the experimental values. The $4 s A_{1}^{\prime}\left\{00^{\circ}\right\}$ and $6 s A_{1}^{\prime}\left\{10^{\circ}\right\}$ diabatic states distribute over 2 or 3 eigenstates, so we listed all these states in the table. For the $4 s A_{1}^{\prime}\left\{00^{0}\right\}, 4 s A_{1}^{\prime}\left\{10^{0}\right\}$ and $4 p E^{\prime}\left\{01^{1}\right\}$ states, the calculated 
TABLE II. Comparison of lifetimes with experiments.

\begin{tabular}{ccc}
\hline \hline State & This work & Experimental value \\
\hline $3 s A_{1}^{\prime}\left\{00^{0}\right\}$ & $39.4 \mathrm{ps}$ & $\sim 1 \mathrm{~ns}^{\mathrm{a}}$ \\
$3 s A_{1}^{\prime}\left\{10^{0}\right\}$ & $11.6 \mathrm{ps}$ & $>66 \mathrm{ps}^{\mathrm{b}}$ \\
$4 s A_{1}^{\prime}\left\{00^{0}\right\}$ & $3.53 \mathrm{ps}$ & $7.6 \mathrm{ps}^{\mathrm{b}}$ \\
& $15.0 \mathrm{ps}$ & \\
$4 s A_{1}^{\prime}\left\{10^{0}\right\}$ & $15.7 \mathrm{ps}$ & $27 \mathrm{ps}^{\mathrm{c}}$ \\
$5 s A_{1}^{\prime}\left\{00^{0}\right\}$ & $329 \mathrm{ps}$ & $3.8 \mathrm{ps}^{\mathrm{b}}$ \\
$5 s A_{1}^{\prime}\left\{10^{0}\right\}$ & $200 \mathrm{ps}$ & \\
$6 s A_{1}^{\prime}\left\{00^{0}\right\}$ & $463 \mathrm{ps}$ & $(1.3 \mathrm{ps})^{\mathrm{d}}$ \\
$6 s A_{1}^{\prime}\left\{10^{0}\right\}$ & $3.15 \mathrm{ps}$ & \\
& $4.93 \mathrm{ps}$ & \\
$7 s A_{1}^{\prime}\left\{00^{0}\right\}$ & $151 \mathrm{ps}$ & $(>5.3 \mathrm{ps})^{\mathrm{d}}$ \\
$7 s A_{1}^{\prime}\left\{10^{0}\right\}$ & $194 \mathrm{ps}$ & $>66 \mathrm{ps}^{\mathrm{b}}$ \\
$3 p E^{\prime}\left\{01^{1}\right\}$ & $132 \mathrm{ps}$ & $21 \mathrm{ps}^{\mathrm{b}}$ \\
$4 p E^{\prime}\left\{01^{1}\right\}$ & $4.58 \mathrm{ps}$ & \\
\hline \hline
\end{tabular}

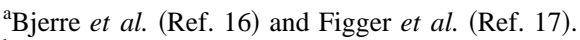

${ }^{b}$ Mistrík et al. (Ref. 8).

${ }^{c}$ Lembo and Helm (Ref. 45).

${ }^{\mathrm{d}}$ Vibrational autoionization (Ref. 45).

lifetimes agree with the experiments within factor of 2 or 3 . But for the $3 s A_{1}^{\prime}\left\{00^{0}\right\}, 5 s A_{1}^{\prime}\left\{00^{0}\right\}$, and $3 p E^{\prime}\left\{01^{1}\right\}$ states, the deviations from the experimental values exceed an order of magnitude. Although the experimental lifetimes for the $3 s A_{1}^{\prime}\left\{00^{0}\right\}$ and $3 p E^{\prime}\left\{01^{1}\right\}$ states are considerably long, the calculations gave much shorter lifetimes for these states. This is because the calculated eigenstates are mixed with $2 s$ states while these should be isolated to explain the experimentally observed long lifetimes. On the contrary, the calculated $5 s A_{1}^{\prime}\left\{00^{0}\right\}$ state is isolated and the resultant lifetime is much longer than the experimental estimate. The mixings of states are governed by the energy differences between diabatic states and if the diabatic energies are modified we will obtain different decay rates. For example, there is an eigenstate with the lifetime of $4.6 \mathrm{ps}$ only $58 \mathrm{~cm}^{-1}$ above the $5 s A_{1}^{\prime}\left\{00^{0}\right\}$ state, which is within the energy difference between the present results and the experiments as listed in Table I, and the $5 s A_{1}^{\prime}\left\{00^{\circ}\right\}$ state can mix with this short lifetime state if the $5 s A_{1}^{\prime}\left\{00^{\circ}\right\}$ diabatic energy is slightly raised. Thus, the discrepancies between the calculations and experiments can be attributed to the errors in the energy levels of diabatic states, since the present model is too crude to reproduce the Rydberg state energies with the accuracy within several $\mathrm{cm}^{-1}$.

As seen from the table, some states with large principal quantum numbers decay within several ps, whereas slow decay rates for small principal quantum numbers are also observed. The fast decay rates of the higher Rydberg states indicate that the indirect predissociation can work efficiently. The irregular distribution of the decay rates with respect to the principal quantum numbers seems to share similar feature with the experiments. The cause of these irregular rates distribution is attributed to the accidental energy matchings between states, because the decay rate and the energy of each state sensitively depends on the energies of the diabatic bases.

In order to examine the energy structure of the Rydberg states, the nearest-neighbor level spacing distribution was

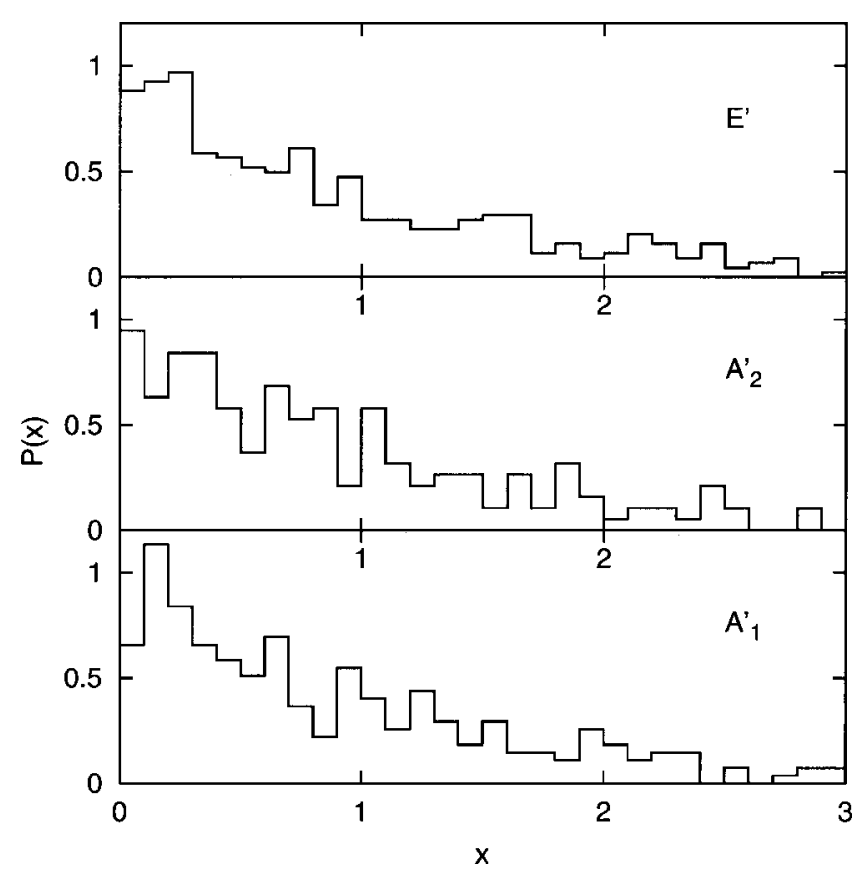

FIG. 11. Normalized level spacing distribution $P(x)$ with $x=\Delta E /\langle\Delta E\rangle$.

obtained for states between $-0.4 \mathrm{eV} \leqslant E \leqslant 0.4 \mathrm{eV}$ and is given in Fig. 11 for each symmetry, $A_{1}^{\prime}, A_{2}^{\prime}$, and $E^{\prime}$, respectively. The number of states in this energy range is 275,191 , and 444 with the mean level spacing $2.88 \times 10^{-3}, 4.16$ $\times 10^{-3}$, and $1.79 \times 10^{-3} \mathrm{eV}$, respectively. As seen from the figure, the level spacings are close to the Poisson rather than the Wigner distribution, which means the states in this energy region are regarded to be regular. Thus some states can dissociate efficiently via mixing with the $2 s A_{1}^{\prime}$ states, whereas other states cannot. We can expect from this distribution that the predissociation rates widely scatter around the mean rate, as observed in the other system ${ }^{46}$ and indeed such behavior is seen in Fig. 10.

There are many states with positive energies in Fig. 10, which are regarded as the resonance states. These states undergo the autoionization through the coupling with the electronic continuum. We included one positive electronic energy diabatic state with the ground vibrational level, which has an overall symmetry of $A_{1}^{\prime}$, to mimic an electronic continuum. The analysis of the eigenstates indicates that the $6 s A_{1}^{\prime}\left\{10^{0}\right\}$ and $7 p E^{\prime}\left\{01^{1}\right\}$ states have large overlap with this positive energy state, suggesting the existence of large autoionization widths for these two states. The photoabsorption intensities to autoionizing Rydberg states have been explored both from experimental and theoretical points of view. ${ }^{20-22}$ In order to compare these results, we need more elaborate treatment of the electronic continuum states, which is beyond the scope of the present calculations.

\section{Predissociation route}

To investigate how the predissociation proceeds, we took the $7 p E^{\prime}\left\{01^{1}\right\}$ base as the initial vector and propagated it for 10 ps. Note that the overall symmetry of this initial state is $E^{\prime}$. In Figs. 12 and 13, the time evolution of the norm $|\Psi(t)|^{2}$ and the mean electronic energy $\left\langle\Psi\left|\hat{H}_{e}\right| \Psi\right\rangle /\langle\Psi \mid \Psi\rangle$ 


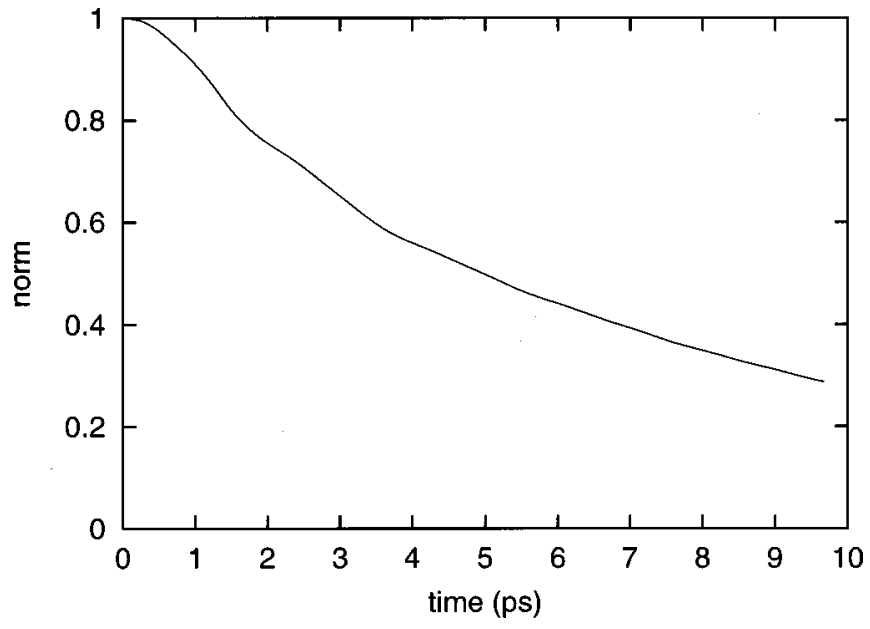

FIG. 12. Norm of the wave packet with an initial state $7 p E^{\prime}\left\{01^{1}\right\}$.

are shown. Figure 14 displays the time evolution of the electronic components of the wave packet, which were obtained by integrating the vibrational components of the wave function. The initial $7 p E^{\prime}\left\{01^{1}\right\}$ state have large overlap, 0.81, with the eigenvector which has the lifetimes of 7.19 ps. The relatively fast decay of the norm observed in Fig. 12 is attributed to this eigenstate. In Fig. 13, we can see a rapid decrease of the mean electronic energy within 1 ps and the corresponding decrease of the effective quantum number from 7.6 to 4.5 . The mean electronic energy oscillates after 1 ps. We can also see this initial decay of 1 ps and the recursive behavior in Fig. 14, where the population of lower Rydberg states gradually increase. The inspection of Fig. 14 and the eigenvector shows that the fast predissociation of the initial $7 p E^{\prime}\left\{01^{1}\right\}$ state is mainly mediated by the transition to the $5 p E^{\prime}, 4 s A_{1}^{\prime}, 3 s A_{1}^{\prime}$, and $3 p E^{\prime}$ state. Although the vibrational states for $5 p E^{\prime}$ and $4 s A_{1}^{\prime}$ state can be specified to be $\left\{02^{2}\right\}$ and $\left\{03^{1}\right\}$, respectively, $3 s A_{1}^{\prime}$ and $3 p E^{\prime}$ electronic state involve several different vibrational states.

The time evolution of the wave packet indicates that the predissociation of Rydberg state with a few vibrational excitation begins with transitions to the lower Rydberg states

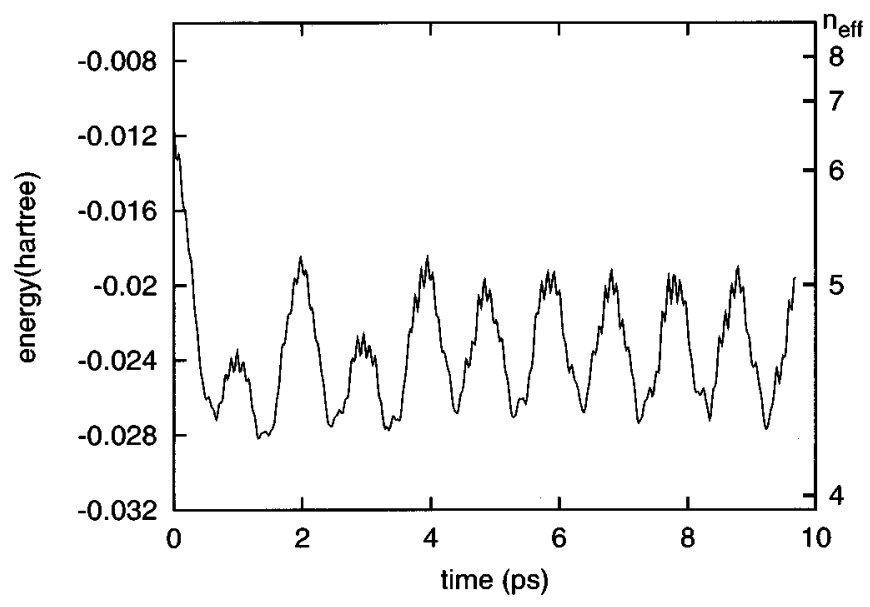

FIG. 13. Mean electronic energy. The initial state is the same as Fig. 12.

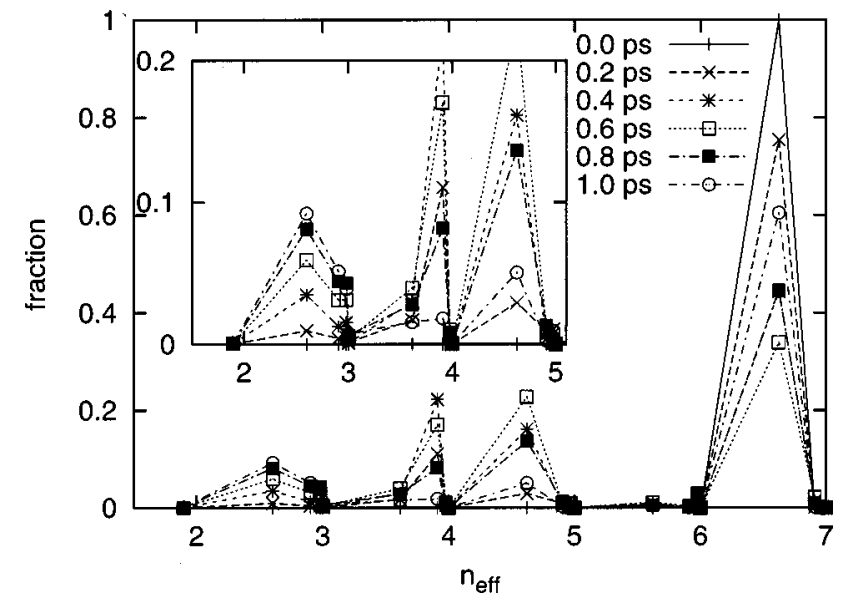

FIG. 14. Time evolution of the electronic components. The initial state is the same as Fig. 12.

with extra vibrational excitations. When these lower intermediate states couples to $n=3$ Rydberg states with high vibrational excitation, the efficient predissociation of the initial state is possible, because these vibrationally excited $n=3$ Rydberg states strongly couple with $2 s$ states having large widths. In this process, the $n p E^{\prime}$ Rydberg states play an important role as intermediate lower Rydberg states, since these states couple strongly with $n=3$ Rydberg states. The $n s A_{1}^{\prime}$ Rydberg states play the role, although their effects of mediation are weaker than those of the $n p E^{\prime}$ states. The $n p E^{\prime}$ Rydberg states have large vibronic couplings with other states because of their Jahn-Teller effect as shown in Fig. 4 and the importance of such effect was emphasized by Stephens and Greene ${ }^{20,21}$ in the context of autoionization and

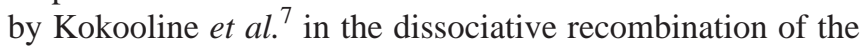
$\mathrm{H}_{3}^{+}$ion.

\section{Implication to the dissociative recombination of $\mathrm{H}_{3}^{+}$}

The dissociative recombination of the $\mathrm{H}_{3}^{+}$ion with an electron begins by the capture of incident electron to generate $\mathrm{H}_{3}$ Rydberg states, with the vibrational excitation of the ion core. If we assume that a low energy incident electron causes a single vibrational excitation to the ion core, the $6 s A_{1}^{\prime}\left\{10^{0}\right\}$ and $7 p E^{\prime}\left\{01^{1}\right\}$ states are the representative candidates for such Rydberg states. These diabatic states have a large overlap with eigenstates having a variety of predissociation lifetimes; 3.15, 4.93, 151 ps for $6 s A_{1}^{\prime}\left\{10^{0}\right\}$ and 7.19, $33.8,151 \mathrm{ps}$ for $7 p E^{\prime}\left\{01^{1}\right\}$ state, which suggests that detailed information about the nonadiabatic transitions among $\mathrm{H}_{3}$ Rydberg states is required to derive the DR rate constant of $\mathrm{H}_{3}^{+}$correctly.

There are many states with the lifetime shorter than 10 ps above $E=0 \mathrm{eV}$ as seen in Fig. 10. Many of these states correspond to vibrationally excited states of the ion core. If multivibrational excitations can take place by the attachment of incident electron, the DR rate becomes faster than that from singly vibrational excited initial state. Even if singly vibrational excited states are prepared by the electron capture, these short lifetime vibrationally excited states can be 
achieved by the vibration-rotation coupling through the Colioris interaction in rotationally excited states. ${ }^{47,48} \mathrm{In}$ addition, the number of accessible states increases by the rotational excitation. ${ }^{47}$ Actually the $\mathrm{H}_{3}^{+}$ion used in the storage ring experiments is estimated to be rotationally excited with the temperature $T_{\text {rot }}=1000-3000 \mathrm{~K} .{ }^{49}$ In this respect, it would be required to treat rotationally excited states in order to interpret the storage ring experiments.

\section{SUMMARY}

The predissociation of $\mathrm{H}_{3}$ Rydberg states were investigated. We paid our attention to the vibrationally excited $2 s$ states and explored the indirect predissociation of higher Rydberg states through $2 s$ electronic state. The effective Hamiltonian was constructed to examine this mechanism, where the $\mathrm{H}_{3}$ system was treated as a Rydberg electron plus $\mathrm{H}_{3}^{+}$ core vibrations. Ab initio calculations of $2 s$ predissociation widths were carried out and these data were incorporated into the effective Hamiltonian. We obtained the complex eigenvalues of this effective Hamiltonian and analyzed the distribution of lifetimes and energies of Rydberg states, and the route of predissociation. Our result shows the irregular lifetime distribution with respect to the principal quantum number which resembles the experiment results, although significant deviation from experiments are observed for some Rydberg states. We found the importance of the accidental energy level matching for fast predissociation and the role of $n p E^{\prime}$ Rydberg states which have strong coupling with the lower Rydberg states. Based on the present results, the implication to the DR process of the $\mathrm{H}_{3}^{+}$ion was given. The importance of rotational excitation was pointed out to resolve the discrepancy between the experiments and the theories.

It is noted that the direct nonadiabatic couplings between $n \geqslant 3$ Rydberg states and $2 p E^{\prime}$ dissociative states were ignored in the present work. Such couplings may be required to obtain more accurate predissociation rates of higher Rydberg states, though the magnitudes of such nonadiabatic coupling elements are considerably smaller than that between the $2 s A_{1}^{\prime}$ and $2 p E^{\prime}$ states. The improvement of the present model, especially the effective potential for a Rydberg electron, will be also needed for the precise decay rate of individual Rydberg states. We employed the local approximation for the effective potential and justified its use by showing that it can reproduce the ab initio Rydberg energies well. However, the local approximation may be not enough as known in the literature of low energy electron-molecular collision. More elaborate treatments of the exchange effect beyond the local approximation may be possible..$^{50,51}$

\section{ACKNOWLEDGMENTS}

We thank Professor T. Oka for calling our attention to the predissociation of $\mathrm{H}_{3}$, and Dr. T. Yamamoto for collaboration at the early stage of this work.

${ }^{1}$ G. Herzberg, J. Chem. Phys. 70, 4806 (1970).

${ }^{2}$ H. F. King and K. Morokuma, J. Chem. Phys. 71, 3213 (1979).
${ }^{3}$ R. N. Porter, R. M. Stevens, and M. Karplus, J. Chem. Phys. 49, 5163 (1968).

${ }^{4}$ I. D. Petsalakis, G. Theodorakopoulos, and J. S. Wright, J. Chem. Phys. 89, 6850 (1988)

${ }^{5}$ Ch. Nager and M. Jungen, Chem. Phys. 70, 189 (1982).

${ }^{6}$ I. F. Schneider, A. E. Orel, and A. Suzor-Weiner, Phys. Rev. Lett. 85, 3785 (2000).

${ }^{7}$ V. Kokoouline, C. H. Greene, and B. D. Esry, Nature (London) 412, 891 (2001).

${ }^{8}$ I. Mistrík, R. Reichle, H. Helm, and U. Müller, Phys. Rev. A 63, 042711 (2001).

${ }^{9}$ J. R. Peterson, P. Devynck, Ch. Hertzler, and W. G. Graham, J. Chem. Phys. 96, 8128 (1992).

${ }^{10}$ P. C. Cosby and H. Helm, Phys. Rev. Lett. 61, 298 (1988).

${ }^{11}$ U. Müller and P. C. Cosby, J. Chem. Phys. 105, 3532 (1996).

${ }^{12}$ U. Müller, U. Majer, R. Reichle, and M. Braun, J. Chem. Phys. 106, 7958 (1997).

${ }^{13}$ U. Müller, Th. Eckert, M. Braun, and H. Helm, Phys. Rev. Lett. 83, 2718 (1999).

${ }^{14}$ U. Müller and P. C. Cosby, Phys. Rev. A 59, 3632 (1999).

${ }^{15}$ I. Dabowski and G. Herzberg, Can. J. Phys. 58, 1238 (2001).

${ }^{16}$ N. Bjerre, I. Hazell, and D. C. Lorents, Chem. Phys. Lett. 181, 301 (1991).

${ }^{17}$ H. Figger, W. Ketterle, and H. Walther, Z. Phys. D: At., Mol. Clusters 13, 129 (1989).

${ }^{18}$ H. Helm, in Dissociative Recombination: Theory, Experiment and Applications, edited by B. R. Rowe, J. B. A. Michell, and A. Canosa (Plenum, New York, 1993), p. 145.

${ }^{19}$ A. Staib and W. Domcke, Z. Phys. D: At., Mol. Clusters 16, 275 (1990).

${ }^{20}$ J. A. Stephens and C. H. Greene, Phys. Rev. Lett. 72, 1624 (1994).

${ }^{21}$ J. A. Stephens and C. H. Greene, J. Chem. Phys. 102, 1579 (1995).

${ }^{22}$ I. Mistrík, R. Reichle, U. Müller, H. Helm, M. Jungen, and J. A. Stephens, Phys. Rev. A 61, 033410 (2000).

${ }^{23}$ A. E. Orel and K. C. Kulander, J. Chem. Phys. 91, 6086 (1989).

${ }^{24}$ J. L. Krause, K. C. Kulander, J. C. Light, and A. E. Orel, J. Chem. Phys. 96, 4283 (1992).

${ }^{25}$ I. F. Schneider and A. E. Orel, J. Chem. Phys. 111, 5873 (1999).

${ }^{26}$ R. Jaquet, W. Cencek, W. Kutzelnigg, and J. Rychlewski, J. Chem. Phys. 108, 2837 (1998).

${ }^{27}$ H. Feshbach, Ann. Phys. (N.Y.) 19, 287 (1962).

${ }^{28}$ R. D. Levine, Quantum Mechanics of Molecular Rate Processes (Clarendon, Oxford, 1969).

${ }^{29}$ C. Eckart, Phys. Rev. 47, 552 (1935).

${ }^{30}$ J. C. Slater, Phys. Rev. 81, 385 (1951).

${ }^{31}$ S. Hara, J. Phys. Soc. Jpn. 22, 710 (1967).

${ }^{32}$ S. Salvini and D. G. Thompson, J. Phys. B 14, 3797 (1981).

${ }^{33}$ J. Schnitker and P. J. Rossky, J. Chem. Phys. 86, 3462 (1987).

${ }^{34}$ E. Fattal, R. Baer, and R. Kosloff, Phys. Rev. E 53, 1217 (1996).

${ }^{35}$ A. J. C. Varandas and H. G. Yu, J. Chem. Soc., Faraday Trans. 93, 819 (1997).

${ }^{36}$ D. T. Colbert and W. H. Miller, J. Chem. Phys. 96, 1982 (1992).

${ }^{37}$ H. G. Yu, Chem. Phys. Lett. 281, 312 (1997).

${ }^{38}$ M. Tashiro and S. Kato, Chem. Phys. Lett. 354, 14 (2002).

${ }^{39}$ MOLPRO is a package of $a b$ initio programs written by H.-J. Werner and P. J. Knowles, with contributions from R. D. Amos, A. Berning, D. L. Cooper et al.

${ }^{40}$ H.-J. Werner and P. J. Knowles, J. Chem. Phys. 82, 5053 (1985).

${ }^{41}$ P. J. Knowles and H.-J. Werner, Chem. Phys. Lett. 115, 259 (1985).

${ }^{42}$ H. Tal-Ezer and R. Kosloff, J. Chem. Phys. 81, 3967 (1984).

${ }^{43}$ A. J. C. Varandas, F. B. Brown, C. A. Mead, D. G. Truhlar, and N. C. Blais, J. Chem. Phys. 86, 6258 (1987).

${ }^{44}$ A. J. Stone, Chem. Phys. Lett. 83, 233 (1981).

${ }^{45}$ L. J. Lembo and H. Helm, Chem. Phys. Lett. 163, 425 (1989).

${ }^{46}$ H. Nakamura and S. Kato, J. Chem. Phys. 110, 9937 (1999).

${ }^{47}$ H. Nakamura and S. Kato, J. Chem. Phys. 112, 1785 (2000).

${ }^{48}$ S. Y. Grebenshchikov, H. Flöthmann, R. Schinke, I. Bezel, C. Wittig, and S. Kato, Chem. Phys. Lett. 285, 410 (1998).

${ }^{49}$ M. J. Jensen, H. B. Pedersen, C. P. Safvan, K. Seiersen, X. Urbain, and L. H. Andersen, Phys. Rev. A 63, 052701 (2001).

${ }^{50}$ F. A. Gianturco, R. R. Lucchese, and N. Sanna, J. Chem. Phys. 102, 5743 (1995).

${ }^{51}$ B. K. Sarpal, K. Pfingst, B. M. Nestmann, and S. D. Peyerimhoff, J. Phys. B 29, 857 (1996). 